\title{
The red sweat of the hippopotamus
}

\section{The red and orange pigments in this secretion account for its protective properties.} ithin a few minutes of perspiration, the colourless, viscous sweat of the hippopotamus gradually turns red, and then brown as the pigment polymerizes. Here we isolate and characterize the pigments responsible for this colour reaction. The unstable red and orange pigments turn out to be non-benzenoid aromatic compounds that are unexpectedly acidic and have antibiotic as well as sunscreen activity.

Although the fluid secreted by the hippopotamus (Hippopotamus amphibius) is not strictly sweat as it is produced by the subdermal glands ${ }^{1}$, it acts like sweat in helping to control body temperature. It is also thought to be antiseptic ${ }^{1}$. We collected this highly alkaline ( $\mathrm{pH} 8.5-10.5$; ref. 1$)$ red secretion by wiping a hippopotamus's face and back with gauze and extracting its chemical constituents with water. (For details of methods, see supplementary information.)

This diluted red solution was carefully concentrated to about $10^{-5} \mathrm{M}$; no further concentration was possible as the solution would have polymerized and turned brown. Subsequent purification by gel filtration and on an ion-exchange resin yielded solutions of two pigments, one red and one orange. We characterized these samples by spectroscopy (ultraviolet spectrum, ${ }^{1} \mathrm{HNMR}$, electrospray ionization and fast-atom bombardment mass spectroscopy). We also converted the labile red pigment into a stable t-butyldimethylsilyl (TBS) derivative and determined its structure by X-ray crystallographic

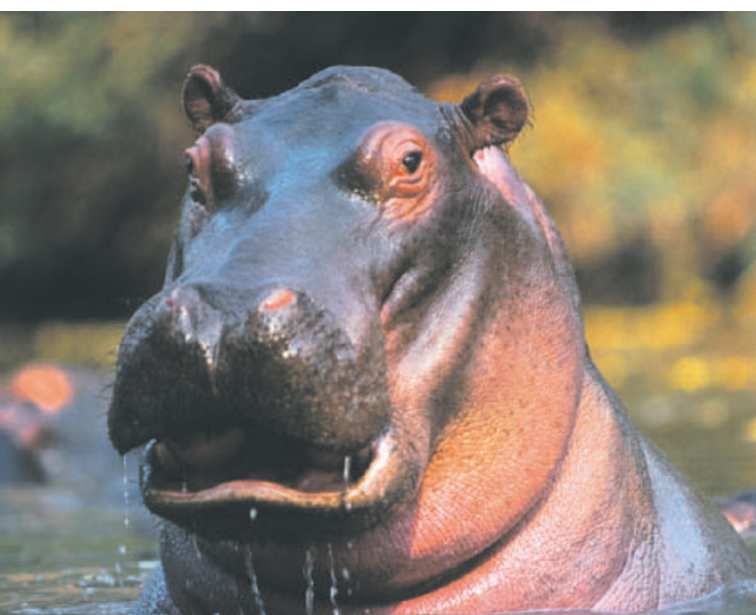

Cool customer: hippos create their own antibiotic sunscreen.

in deuterated chloroform indicates that the structure is symmetrical and that the hydroxy proton of the enol group forms hydrogen bonds with the oxygen atoms at positions 4 and 5 (Fig. 1,4a) to give a mesomeric diketonate; alternatively, $\mathbf{4}$ may exist as a mixture of two tautomers.

The $\mathrm{p} K_{\mathrm{a}}$ value of 4 in $50 \%$ aqueous methanol was measured as about 2.7-3.3; under the same conditions, the $\mathrm{p} K_{\mathrm{a}}$ values of acetic acid and phenol are 5.6 and 11.8, respectively. This indicates that $\mathbf{4}$ is an unexpectedly strong acid that should exist predominantlyin its dissociated form ( 5 in Fig. 1 ) in water.

On the basis of their structural similarity to 4 , the natural pigments

analysis (Fig. 1, compound 1).

From these results, the structures of the red and orange pigments were deduced (Fig. 1, compounds 2 and 3 , respectively). We named the red pigment 'hipposudoric acid' and the orange pigment 'norhipposudoric acid'. The framework of $\mathbf{2}$ and $\mathbf{3}$ is probably biochemically derived by oxidative dimerization of homogentisic acid, a metabolite of the amino acids phenylalanine and tyrosine.

The tautomeric or resonant structures of the highly hydrophilic 2 and 3 were studied by using a model compound 4 , which shares a chromophore with these pigments and is soluble in organic solvents. Compound 4 was prepared by oxidation of the corresponding tetrahydrodiquinone in deuterated chloroform.

Analysis of the ${ }^{1} \mathrm{H}$ NMR spectrum of 4<smiles></smiles><smiles>O=C(O)Cc1cc(=O)c2c(C(=O)O)c3c(=O)ccc(O)c-3c=2c1=O</smiles><smiles>O=C1C=CC(=O)c2cc(=O)ccc1c2O</smiles>

4

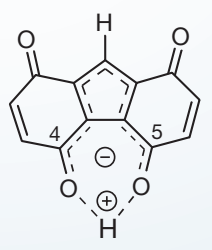

$4 a$
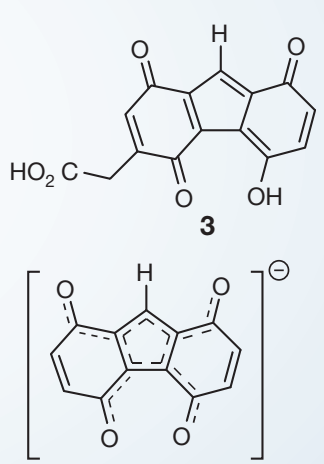

5

Figure 1 Structures of the red and orange pigments isolated from hippopotamus sweat. Compound $\mathbf{1}$ is the $t$-butyldimethylsilyl (TBS) derivative of the labile red pigment hipposudoric acid (compound 2) used for X-ray crystallography; asterisk indicates a racemic carbon; compound $\mathbf{3}$ is the orange pigment norhipposudoric acid. Compound $\mathbf{4}$ is the synthesized model pigment; $\mathbf{4 a}$ is the coordinated hydrogenbonded form; and $\mathbf{5}$ is the anionic structure of the model, which resembles the forms of the pigments that exist above about pH3.

$\mathbf{2}$ and $\mathbf{3}$ are also likely to exist in their anionic forms in aqueous solution and in the hippopotamus's alkaline sweat, their charge delocalized by resonance. The anions are probably stabilized by intermolecular hydrogen bonding with the solvent.

What is the function of these pigments as far as the hippopotamus is concerned? Their spectra in the ultraviolet/visible range (200-600 nm; see supplementary information) indicate that they may act as sunscreens. The red pigment 2 also has antibiotic activity: at concentrations lower than that found on the hippopotamus's skin, it inhibits the growth of the pathogenic bacteria Pseudomonas aeruginosa A3 and Klebsiella pneumoniae (see supplementary information).

The isolated pigments are highly unstable, but when they dry on the skin surface in the presence of mucus they maintain their colour for several hours before they polymerize into brown solids. The pigment precursors remain to be identified and the stabilizing effect of the mucus has yet to be investigated.

Yoko Saikawa $^{\star}$, Kimiko Hashimoto ${ }^{\star} \dagger$, Masaya Nakata*, Masato Yoshihara $\ddagger$, Kiyoshi Nagai $\neq$, Motoyasu Ida

Teruyuki Komiya

${ }^{\star}$ Department of Applied Chemistry, Faculty of Science and Technology, Keio University, 3-14-1

Hiyoshi, Kohoku-ku, Yokohama 223-8522, Japan $\dagger$ Present address: Kyoto Pharmaceutical University, 1 Shichono-cho, Misasagi, Yamashina-ku, Kyoto 607-8412, Japan

e-mail:kimikoh@mb.kyoto-phu.ac.jp

‡Ueno Zoological Gardens, 9-83 Ueno Kouen,

Taitou-ku, Tokyo 110-8711, Japan

1. Eltringham, S. K. The Hippos 8-38 (Poyser Natural History Series, London, 1999).

Supplementary information accompanies this communication on Nature's website.

Competing financial interests: declared none. 